\title{
Molecular Tumor Markers as a Tool for Diagnosis, Prognosis, Prediction and Therapy of Tumors
}

\section{Hussein Abd Elhay Kaoud* \\ Full Professor, Cairo University, Egypt}

Submission: August 21, 2017; Published: November 22, 2017

*Corresponding author: Hussein Abd Elhay Kaoud, Full Professor, Cairo University, Giza, Egypt, Email: ka-oud@link.net

Abstract

Molecular tumor markers can be produced directly by tumor or non tumor cells as a response to the presence of a tumor. Most tumor markers are tumor antigens but not all tumor antigens can be used as tumor markers. Molecular markers are diagnostic, prognostic or predictive. The article discussed the Molecular tumor markers; significance of each markers and its role in diagnosis, prognosis and prediction of tumor and tests used (Figure 1).

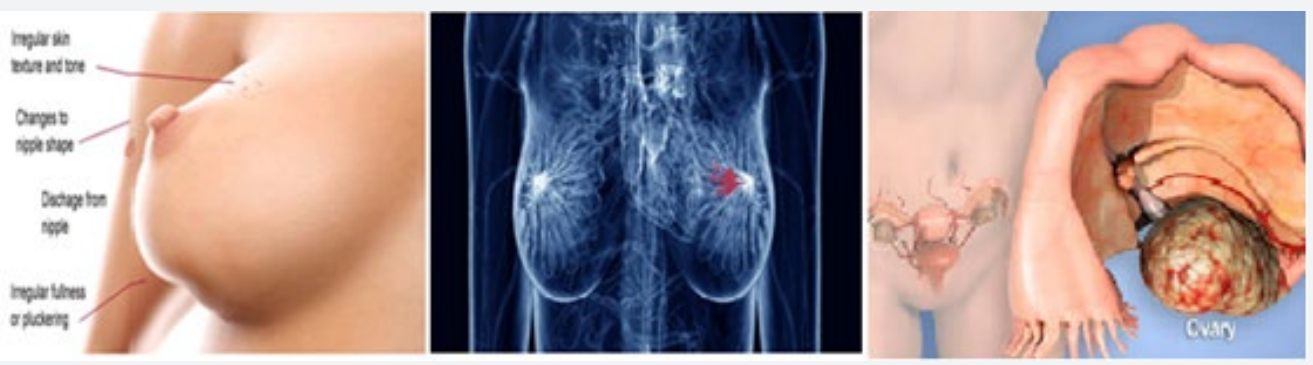

Figure 1: Molecular tumor markers as a tool for diagnosis, prognosis, prediction and therapy of tumors.

Novel molecular markers are being developed with the increasing understanding of the molecular environment of the cancer cell and other pathological cells. Most tumor markers are tumor antigens, but not all tumor antigens can be used as tumor markers. Biomarkers can determine the appropriate dose in the early stages of a new antidepressant medication for cancer clinical development. They also help determine who to treat, how aggressively to treat and which candidates are likely to respond to a particular drug, and the dose is most effective. Theranostic, use a combination of diagnosis and therapy sessions, and can be different perception. Imaging can be used to track drug delivery within the body. However, imaging can also be used to activate the drug release from the outside, by an external stimulus. These external influences to be laser light, temperature or ultrasound, for example.

Keywords: Cancer; Molecular markers; Monitoring; Theranostic

\section{Introduction}

Cancer is a multigene disease (cluster of diseases) which arises as a result of mutational \& this contributes to development of cancer genotype. It involves alteration of three main classes of genes:
1) Proto-Oncogenes
2) Tumor suppressor genes
3) DNA repair genes.

Epigenetic changes coupled with activation of complex signaling networks. And these alterations resist the naturaluphenotype \& inherent death mechanisms embedded in cells (apoptosis) coupled with deregulation of cell proliferation events. These genetic alterations include gene rearrangements, point mutations and gene amplifications leading to disturbances in molecular pathways regulating cell growth, survival and can also be used for detection. When these changes manifest in majority of patients with specific type of tumor this can be 
used as tumor markers (Biomarkers). Metastasis and developing targeted therapies besides predicting responses to treatment. Molecular markers may be diagnostic, prognostic or predictive.

A tumor biomarker should be indicative of: Tumor susceptibility of the patient- Severity or virulence of the tumorPrognosis of the disease- Tumor burden (Including metastasis). They include a broad range of biochemical entities such as: Nucleic acids, Proteins, Sugars, Lipids, Small metabolites, Cytogenetic and cytokinetic parameters, Whole tumor cells and Cancer stem cells.

\section{Methodology}

\section{Classification of molecular tumor biomarkers}

On the basis of their chemical nature tumor markers can be proteins, conjugated proteins, peptides or carbohydrates. Proteins or conjugated proteins may be enzymes, hormones or fragments of proteins and Sequencing of genes. Tumor markers are usually classified into three categories: prognostic, predictive, and pharmacodynamic [1] (Figure 2).

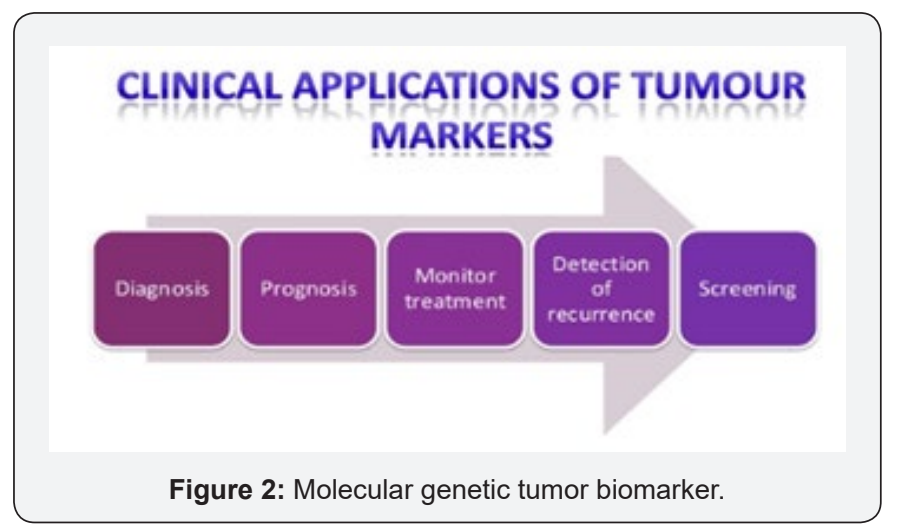

\section{Genetic tumor markers result from}

a) Deviations from diploid chromosomes to hypo and hyper diploidy as well as aneuploidy noted in malignant tumors.

b) Enhanced cell proliferation, most important hall mark of cancer can be assessed by flow cytometric analysis of DNA content.

c) Gene deletions can be discovered by PCR using microsatellite probes to various chromosomes and sites.

d) Oncogenes are derived from proto-oncogenes that may be activated by dominant mutations, insertions, deletions, translocations, or inversions. Most oncogenes are associated with hematologic malignancies, such as leukaemia and to lesser extent solid tumors.

\section{The most common genetic markers include}

BRCA ashkenazi Jewish mutations: BRCA1 and BRCA2 are human genes that belong to a class of genes known as tumor suppressors. Mutation of these genes has been linked to hereditary breast and ovarian cancer. Genetic tests are available to check for BRCA1 and BRCA2 mutations a blood sample is required for these tests.

B-cell gene rearrangement: The $\mathrm{B}$ - and T-cell rearrangement test can detect a monoclonal population of B- and T-cells. Strongly indicative of neoplasia. This is accomplished through the use of DNA probes.

BCL-2 gene rearrangement: Gene Rearrangement bcl-2; Major Breakpoint Region (MBR); minor cluster region (mcr); $\mathrm{t}$ (14:18) Translocation. Applies to Follicular B-Cell Lymphomas. A translocation between immunoglobulin genes (heavy chain or light chain genes) and bcl-2 results in the over expression of bcl2 protein and thus the expansion of $B$ cells due to inhibition of cell death.

BCR-ABL gene rearrangement: Translocation bcr/abl; Gene Rearrangement bcr; Philadelphia Chromosome; t (9:22) Applies to Acute Myelogenous Leukemia (ALL); Chronic Myelogenous Leukemia (CML).

BRAF gene mutation detection

c-kit mutation detection for systemic mastocytosis

KRAS gene mutation detection

Muir Torre syndrome [MTS]

PMLRAR alpha t $(15 ; 17)$ translocation: Breast cancer two genomic: assays are currently in use for breast cancer: Oncotype DX and Mamma Print. Oncotype DX looks at the activity of 21 genes, Mamma Print at 70 genes. A number of genetic alterations and consequent metabolic changes are involved in metastatic progression of cancer [2].

\section{Molecular proteins and conjugated protein markers (products of tumors)}

\section{Oncofetal antigens}

Alpha-fetoprotein: Alpha-fetoprotein (AFP, $\alpha$-fetoprotein; also sometimes called alpha-1-fetoprotein, alpha-fetoglobulin, or alpha fetal protein) is a protein $[3,4]$, that in humans is encoded by the AFP gene [5]. The AFP gene is located on the q arm of chromosome 4 (4q25).

AFP is measured in pregnant women through the analysis of maternal blood or amniotic fluid, as a screening test for a subset of developmental abnormalities. Some of the diseases in which AFP will be elevated in a person are listed below:

a) Hepatocellular carcinoma/hepatoma: $\uparrow \alpha$-fetoprotein.

b) Neural tube defects: $\uparrow \alpha$-fetoprotein in amniotic fluid and maternal.

Carcinogenic embryonic antigen: Borderline: 25ng $\backslash \mathrm{ml}-$ $5 \mathrm{ng} \backslash \mathrm{ml}$ (testing immunoassay). Elevated\} $5 \mathrm{ng} \backslash \mathrm{ml}$. Carcinogenic colon, pancreas, lung, breast and ovary. $75 \%$ of the patients with recurrent colorectal cancers elevate CEA before the development 
of the symptoms. Gastrointestinal cancer, cervix cancer, lung cancer, ovarian cancer, breast cancer, urinary tract cancer [6].

\section{Tumor associated protein}

CA15-3 (Cancer Antigen 15-3): Is a tumor marker used to monitor certain cancers, especially breast cancer.

CA 27-29: A tumor marker similar to the CA 15-3 that is found in the blood of most breast cancer patients may be used to check for recurrence in women previously treated for stage II and stage III breast cancer. And can also be elevated by cancers of the colon, stomach, kidney, lung, ovary, pancreas, uterus, and liver.

CA19-9 (carbohydrate antigen 19-9, also called cancer antigen 19-9 or sialylated Lewis (a) antigen)

CA-125 (cancer antigen 125, carcinoma antigen 125, or carbohydrate antigen 125): Also known as mucin 16, has found application as a tumor marker or biomarker that may be elevated in the blood of some patients with specific types of cancers, or other benign conditions.

Early Prostate Cancer Antigen (EPCA): Is a nuclear matrix protein linked with the nuclear transformations that occur in early prostate cancer development [7].

PCA3/DD3: The prostate cancer antigen 3 (PCA3 or DD3) gene encodes a prostate-specific mRNA that is over expressed in cancer prostate tissue [8].

Ki 67 antige: Is a labile, non-histone nuclear protein that is tightly linked to the cell cycle. Ki-67 score is the most often measured on histological sections by IHC methodology and is defined as the percentage of stained invasive carcinoma cells $[9,10]$.

Tumor protein p53: Is involved in several critical pathways including cell cycle arrest, apoptosis, DNA repair, and cellular senescence. In breast cancer, approximately $30 \%$ of patients display TP53 gene mutation, but this frequency fluctuates from more than $80 \%$ in basal-like to less than $15 \%$ in luminal-A subtypes [11].

Chromogranin A (CGA or GRN-A): Part of the granin family of proteins, is an acidic protein that has been identified in all neuroendocrine cell types, it has been examined for its diagnostic and prognostic values as a biomarker for prostate cancer [12].

Carcinogenic antigen: Cancer cells may elicit the production of antibodies against self-antigens or against neo-antigens $[13,14]$. Thus, the 'immunosignature' is defined as the common pattern of binding that is shared by patients with a given disease but not with another disease or with healthy controls [15].

B7-H3: B7-H3 is the first immune molecule that possibly participates in the development of prostate cancer and in predicting the recurrence and progression of cancer.

\section{Molecular hormonal markers}

Molecular hormonal receptors (HER-2 oncogene, Ki67, and p53 proteins): The hormone receptors are expressed proteins both in the epithelium and in breast stroma which bind to circulating hormones, mediating their cellular effects [16].

HER-2 \neu: (Human Epidermal Growth Factor Receptor 2) is a marker for breast cancer. HER2-positive breast cancer is a breast cancer that tests positive for a protein called human epidermal growth factor receptor 2 (HER2), which promotes the growth of cancer cells.

The HR: The HR best studied in breast cancer is estrogen receptor (ER) and progesterone receptors (PR). Breast cancers classified by positive immune-histochemistry (IHC) expression of ER and PR has different clinical, pathological, and molecular characteristics [17].

Calcitonin: Tumor marker for medullary carcinoma thyroid. It is 32 amino-acids linear polypeptide hormone. Produced by para-follicular cells of the thyroid. Normal level: male $=3-26 n g \backslash$ $\mathrm{L}$, Female $=2-17 \mathrm{ng} \backslash \mathrm{L}$. Also elevated with other cancers, such as lung cancers and leukemias, but not used to detect them.

a-Methylacyl Coenzyme A Racemase (AMACR): It is an, in prostate cancer [18-20].

TMPRSS2-ERG gene fusion rearrangement: Transmembrane protease serine 2 , play a possible role in prostate tumor metastasis through the activation of protease-activated receptor-2 (PAR-2) [21].

\section{Molecular enzyme markers}

\section{Prostate-specific antigen (PSA)}

Protein tyrosine phosphatase: Receptor type, $\mathrm{C}$ also known as PTPRC is an enzyme that, in humans, is encoded by the PTPRC gene. [20] PTPRC is also known as CD45 antigen (CD stands for cluster of differentiation), which was originally called leukocyte common antigen (LCA).

Tumor M2-PK: Can be elevated in many tumor types, rather than being an organ-specific tumor marker such as PSA. Increased stool (fecal) levels are being investigated as a method of screening for colorectal tumors, and EDTA plasma levels are undergoing testing for possible application in the follow-up of various cancers.

Telomerase: One of the best markers for human cancer associated with only malignant tumors. Telomerase enzyme ensures the maintenance of telomere and thereby protecting the cell from degradation and death. In cancer the Telomerase shuttling system is impaired. TRAP (telomeric repeat amplification protocol) assay is used for the detection of its activity. GRN163l (drug) and GRNAV1 is a vaccine. 


\section{miRNAs}

MicroRNAs are naturally occurring, noncoding small RNA molecules of 21-24 nucleotides that binds partially or completely to 3'untranslated regions (3'-UTRs) of protein-coding genes, leading to cleavage or translational repression of targets [19]. The miRNA can be stably present in whole blood, serum, and plasma, but the origin of circulating miRNA is still unclear. It has been proposed that tumor-associated miRNAs can be released into bloodstream when tumor cells are dying and being lysed or through active secretion of miRNA loaded exosomes by tumor cells $[20,21]$.

\section{Therapeutic Biomarkers}

Biomarkers for cancer have multiple effects in cancer intervention. Reliable biomarker can be used for cancer diagnosis and evaluation of risks and diagnostics, and to monitor the effectiveness of treatment. More importantly, some, but not all, biomarkers can be used as targets therapeutic. This is because some of the biomarkers may be a mere "messengers"that do not contribute directly to the growth of the tumor and therefore are not ideal therapeutic targets.

a) Histone deacetylases (HDACs)

b) Mammalian target of rapamycin or FK506-binding protein 12-rapamycin-associated protein 1 .

\section{New Biomarkers}

\section{Adiponectin and leptin in breast cancer}

Leptin mRNA expression in is higher in cancer cells so Leptin induces proliferation of cancer cells. Adiponectin inhibit proliferation of cancer cells.

\section{Adhesion molecules and metastasis}

\section{Receptor C-X-C chemokine Receptor Type 4 (CXCR4)}

This receptor is required for migration of breast cancer cells from the primary site to lung, bones, and lymph nodes, which represent organs that secrete high levels of chemokine CXCL12. For this reason, CXCR4 has been found to be a prognostic marker in breast cancer, among other types of cancer.

\section{Conclusion}

Tumor markers can be produced directly by the tumor or by non-tumor cells as a response to the presence of a tumor. Most tumor markers are tumor antigens, but not all tumor antigens can be used as tumor markers. Usually classified into three categories: prognostic, predictive, and pharmacodynamic. A better understanding of the mechanisms behind biomarker elevation in biological fluids may facilitate the discovery of new tumor markers. Current cancer biomarkers suffer from low diagnostic sensitivity and specificity and have not yet made a major impact in reducing cancer burden. New tumor markers must undergo rigorous validation before they are introduced into routine clinical care. Theranostics take advantage of these abundant molecular targets to gain specific access to tumors, visualizing the site of disease, defining the presence or absence of a molecular target, and delivering cytotoxic payloads directly to tumor sites. Study of tumor markers that include current biomarkers or examination of fluids and tissues that are in close proximity to the tumor might also assist in identification of novel tumor markers.

\section{References}

1. Sawyers CL (2008) The cancer biomarker problem. Nature 452(7187): 548-552.

2. Torre D (1968) Multiple sebaceous tumors. Arch Dermatol 98(5): 549551.

3. Yun J, Johnson JL, Hanigan CL, Locasale JW (2012) Interactions between epigenetics and metabolism in cancers. Front Oncol 15(2): 163.

4. Taylor DD, Gercel-Taylor C (2008) MicroRNA signatures of tumorderived exosomes as diagnostic biomarkers of ovarian cancer. Gynecol Oncol 110(1): 13-21.

5. Chin LJ, Slack FJ (2008) Truth serums for cancer microRNAs have major potential as cancer biomarkers. Cell Research 18(10): 983-984.

6. Mitchell PS, Parkin RK, Kroh EM, Fritz BR, Wyman KS, et al. (2008) Circulating microRNAs as stable blood-based markers for cancer detection. Proceedings of the national academy of sciences of the United States of America 105(30): 10513-10518.

7. Casciato DA, Territo MC (2008) Manual of clinical oncology. In: Casciato DA, Territo MC (Eds.), Spiral manual of clinical oncology lippincott manual series, $\left(6^{\text {th }}\right.$ edn), Lippincott Williams \& Wilkins, USA.

8. Uetsuki H, Tsunemori H, Taoka R, Haba R, Ishikawa M, et al. (2005) Expression of a novel biomarker, EPCA, in adenocarcinomas and precancerous lesions in the prostate. J Urol 174(2): 514-518.

9. Kirby RS, Fitzpatrick JM, Irani J (2009) Prostate cancer diagnosis in the new millennium: strengths and weaknesses of prostate-specific antigen and the discovery and clinical evaluation of prostate cancer gene 3 (PCA3). BJU Int 103(4): 441-445.

10. Gerdes J, Lemke H, Baisch H, Wacker HH, Schwab U, et al. (1984) Cell cycle analysis of a cell proliferation associated human nuclear antigen defined by the monoclonal antibody Ki-67. J Immunol 133(4): 17101715.

11. Reyal F, Bollet MA, Caly M, Gentien D, Carpentier S, et al. (2012) Respective prognostic value of genomic grade and histological proliferation markers in early stage ( $\mathrm{pN} 0$ ) breast carcinoma. PLoS ONE 7(4): e35184.

12. Sørliea T, Charles M, Robert P, Turid Aasf T, Geislerg S, et al. (2001) Gene expression patterns of breast carcinomas distinguish tumor subclasses with clinical implications. Proc Natl Acad Sci USA 98(19): 10869-10874.

13. Deftos LJ (1998) Granin-A parathyroid hormone-related protein, and calcitonin gene products in neuroendocrine prostate cancer. Prostate Suppl 8: 23-31.

14. Stafford P, Halperin RF, Legutki JB, Magee DM, Galgiani J, et al. (2012) Physical characterization of the immunosignaturing effect. Mol Cell Proteomics 11(4): M111.011593.

15. Stafford P, Johnston S (2011) Microarray technology displays the complexities of the humoral immune response. Expert Review of Molecular Diagnostics 11(1): 5-8. 
16. Chase BA, Johnston SA, Legutki JB (2012) Evaluation of biological sample preparation for immunosignature based diagnostics. Clinical and Vaccine Immunology 19(3): 352-358.

17. Haslam SZ (1989) The ontogeny of mouse mammary gland responsiveness to ovarian steroidhormones. Endocrinology 125(5): 2766-2772.

18. Mukherjee D, Zhao J (2013) The Role of chemokine receptor CXCR4 in breast cancer metastasis. Am J Cancer Res 3(1): 46-57.
19. Zha S, Ferdinandusse S, Denis S, Wanders RJ, Ewing CM, et al. (2003) Alpha-methylacyl-CoA racemase as an androgen-independent growth modifier in prostate cancer. Cancer Res 63(21): 7365-7376.

20. Wilson S, Greer B, Hooper J (2005) The membrane-anchored serine protease, TMPRSS2, activates PAR-2 in prostate cancer cells. Biochem J 388(3): 967-972.

21. Slack JMW (2014) Genes-A very short introduction. Oxford University Press, UK.
This work is licensed under Creative Commons Attribution 4.0 License

DOI: $10.19080 /$ JTMP.2017.02.555588
Your next submission with Juniper Publishers will reach you the below assets

- Quality Editorial service

- Swift Peer Review

- Reprints availability

- E-prints Service

- Manuscript Podcast for convenient understanding

- Global attainment for your research

- Manuscript accessibility in different formats ( Pdf, E-pub, Full Text, Audio)

- Unceasing customer service

Track the below URL for one-step submission https://juniperpublishers.com/online-submission.php 